\title{
Correction to: Value of pre- and intraoperative diagnostic methods in suspected glottic neoplasia
}

\author{
Camilla Slot Mehlum ${ }^{1}\left[\right.$ - Thomas Kjaergaard ${ }^{2} \cdot$ Ågot Møller Grøntved $^{1} \cdot$ Nina Munk Lyhne ${ }^{3}$. \\ Andreas Peter Schjellerup Jørkov ${ }^{4}$. Preben Homøe ${ }^{4}$. Jesper Filtenborg Tvedskov ${ }^{5}$ Kristian Hveysel Bork ${ }^{5}$. \\ Sören Möller ${ }^{6}$. Gita Jørgensen ${ }^{1}$ • Bahareh Bakhshaie Philipsen ${ }^{1} \cdot$ Christian Godballe $^{1}$
}

Published online: 6 May 2020

○) Springer-Verlag GmbH Germany, part of Springer Nature 2020

\section{Correction to: \\ European Archives of Oto-Rhino-Laryngology (2020) 277:207-215 \\ https://doi.org/10.1007/s00405-019-05698-w}

As authors of the article Value of pre-and intraoperative diagnostic methods in suspected glottic neoplasia [1] we would like to draw attention to two unfortunate errors that occurred in the paper.

In a nationwide prospective cohort study of 261 patients suspected for glottic neoplasia, we evaluated the individual and combined ability of videostroboscopy (VS), high-speed digital imaging (HSDI), enhanced endoscopy (EE) and saline infusion (SI) to predict neoplasia.

In Table 4 Logistic regression modelling of relevant explanatory variables in predicting neoplasia, the Odds Ratio (OR) for Male gender is in fact the OR for Female gender and thus reflects the reduced OR in females. The conclusion of the paper is correct in that significant association $(p<0.05)$ only appears between age, male gender, and perpendicular vasculature in intraoperative $\mathrm{EE}$, and the endpoint neoplasia. However, the published Table 4 would mislead the reader to the interpretation that the odds for neoplasia were reduced in males. We include the corrected Table 4, so that readers will be in no doubt about the impact of gender in glottic neoplasia.

A minor error that escaped our attention in the manuscript is the number of SI. We investigated 237 SI, as correctly shown in Table 2, and not 234 as described in the result section.

We deeply regret and apologize for these errors.

Another issue that is not erroneous, but may need clarification is on the number of VS. We investigated 188 VS, as correctly stated in the result section, but 19 datasets did not provide enough details on the mucosal wave, and thus 169 VS were further analyzed, as correctly shown in Table 2.
The original article can be found online at https://doi.org/10.1007/ s00405-019-05698-w.

\section{Camilla Slot Mehlum}

Camilla.mehlum@rsyd.dk

1 Department of ORL Head and Neck Surgery and Audiology, Odense University Hospital, J. B. Winsløwsvej 4, 5000 Odense, Denmark

2 Department of Otorhinolaryngology-Head and Neck Surgery, Aarhus University Hospital, Palle Juul-Jensens Boulevard 165, 8200 Aarhus N, Denmark

3 Department of Head and Neck Surgery, Aalborg University Hospital, Hobrovej 18-22, 9000 Aalborg, Denmark
4 Department of Otorhinolaryngology and Maxillofacial Surgery, Zealand University Hospital, Lykkebækvej 1, 4600 Køge, Denmark

5 Department of Otorhinolaryngology, Head and Neck Surgery, and Audiology, Rigshospitalet, University Hospital of Copenhagen and University of Copenhagen, Blegdamsvej 9, 2100 Copenhagen, Denmark

6 OPEN-Open Patient Data Explorative Network and Department of Clinical Research, Odense University Hospital and University of Southern Denmark, J. B. Winsløwsvej 9, 5000 Odense, Denmark 
Table 4 Logistic regression modelling of relevant explanatory variables in predicting neoplasia

\begin{tabular}{|c|c|c|c|c|c|}
\hline & \multicolumn{2}{|c|}{ Complete case analysis $(n=68)$} & \multicolumn{3}{|c|}{ Analysis with imputation $(n=261)$} \\
\hline & Odds ratio $(95 \% \mathrm{CI})$ & $P$ value & $\begin{array}{l}\text { Number of imputed } \\
\text { observations }\end{array}$ & Odds ratio $(95 \% \mathrm{CI})$ & $P$ value \\
\hline Age & $1.04(0.98-1.11)$ & 0.191 & 0 & $1.04(1.01-1.07)$ & 0.007 \\
\hline Male gender & $6.25(1.52-25.0)$ & 0.012 & 0 & $4.55(2.27-9.09)$ & 0.000 \\
\hline \multicolumn{6}{|l|}{ Smoking } \\
\hline Never & (Empty) & & - & - & - \\
\hline Previously $^{\mathrm{a}}$ & $1.16(0.26-5.24)$ & 0.844 & 14 & $0.73(0.19-2.83)$ & 0.653 \\
\hline Currently & $-{ }^{\mathrm{b}}$ & & 14 & $0.70(0.19-2.54)$ & 0.586 \\
\hline Impaired/absent mucosal wave in VS & $3.12(0.74-13.12)$ & 0.120 & 92 & $1.84(0.83-4.07)$ & 0.133 \\
\hline Perpendicular vessels in preoperative EE & $0.64(0.13-3.24)$ & 0.592 & 161 & $0.73(0.30-1.79)$ & 0.498 \\
\hline Perpendicular vessels in intra-operative EE & $4.98(0.73-33.90)$ & 0.101 & 52 & $2.56(1.04-6.31)$ & 0.041 \\
\hline Partly or complete mucosal fixation in SI & $0.61(0.07-5.38)$ & 0.655 & 24 & $1.51(0.58-3.88)$ & 0.397 \\
\hline Baseline odds & $0.05(0.00-2.84)$ & 0.145 & & $0.72(0.06-8.49)$ & 0.797 \\
\hline
\end{tabular}

Estimates of odds ratios for neoplasia are presented with and without imputation for handling missing data by use of a Multivariate Imputation by Chained Equations model

HSDI was omitted because of few observations almost all from one center

$V S$ videostroboscopy, $E E$ enhanced endoscopy, $S I$ saline infusion, $C I$ confidence interval

${ }^{\text {a }}$ Smoking cessation $>3$ months ago

${ }^{\mathrm{b}}$ Omitted because of collinearity

\section{Reference}

1. Mehlum CS, Kjaergaard T, Grontved AM, Lyhne NM, Jorkov APS, Homoe P, Tvedskov JF, Bork KH, Moller S, Jorgensen G, Philipsen BB, Godballe C (2019) Value of pre- and intraoperative diagnostic methods in suspected glottic neoplasia. Eur Arch Otorhino-laryngol. https://doi.org/10.1007/s00405-019-05698-w

Publisher's Note Springer Nature remains neutral with regard to jurisdictional claims in published maps and institutional affiliations. 